\title{
Surgical Excision of Painful Fibular Sesamoid: A case report
}

\author{
by Taren Cardona, BS ${ }^{1} \rrbracket$, Al Kline, DPM $^{2} \bowtie$
}

The Foot \& Ankle Journal 1 (8): 2

A case report describing the removal of a painful fibular sesamoid is described. This case represents a comminuted fibular sesamoid fracture with radiographic evidence of avascular necrosis. After failed attempts at conservative treatments, the surgical approach, postoperative treatment and prognosis is discussed. Unlike the tibial sesamoid, excision of the fibular sesamoid appears to have a better longterm prognosis.

Key words: Fibular sesamoid fracture

Accepted: July 2008

Published: August 2008

This is an Open Access article distributed under the terms of the Creative Commons Attribution License. It permits unrestricted use, distribution, and reproduction in any medium, provided the original work is properly cited. @The Foot \& Ankle Journal (www.faoj.org)

Surgical resection of a sesamoid was originally described in 1904 by Inge. ${ }^{3}$ There are several etiologies of sesamoid pain. The most commonly encountered are avascualr necrosis, symptomatic bipartite sesamoid, fracture/trauma, osteomyelitis, or osteoarthritis. ${ }^{10}$ Surgery is usually reserved until all conservative treatment has been exhausted and there is continued pain under the sesamoids.

Tibial sesamoid injury is more common since this sesamoid bears a greater percentage of body weight in the gait cycle. ${ }^{10}$ Surgical resection of the fibular sesamoid has less potential for pain and swelling after surgery and therefore a shorter recovery period than tibial sesamoid excision. ${ }^{10}$

\footnotetext{
Address correspondence to: Al Kline, DPM

3130 South Alameda, Corpus Christi, Texas 78404.

${ }^{1}$ Submitted during clinical clerkship in May 2008, Corpus Christi, Texas 78404. Fourth year podiatry student, Barry University School of Podiatric Medicine.

2 Adjunct Clinical Faculty, Barry University School of Podiatric Medicine. Private practice, Chief of Podiatry, Doctors Regional Medical Center. Corpus Christi, Texas, 78411.
}

We report on a case of fibular sesamoid arthritis refractory to conservative treatment and subsequent resection of the fibular sesamoid.

\section{Case Report}

A 54 year old female presents with a chief complaint of "pain and swelling" to plantar region of the left first and second metatarsophalangeal joints. The condition started after walking extensively and has persisted for two years. Prior conservative treatment includes injection therapy, immobilization with CAM walker, and orthotics. Radiographs reveal a comminuted fibular sesasmoid fracture and arhtiritis. (Fig.1) MRI report was unavailable for review. Surgical fibular sesamoid excision was agreed upon since all conservative measures had been exhausted. The patient's medical history includes hypertension and breast cancer. Physical exam reveals mild hallux valgus with edema to the left first and second metatarsophalangeal joints plantarly with positive pain on palpation and pain upon extension of the first and second metatarsophalangeal joints. 


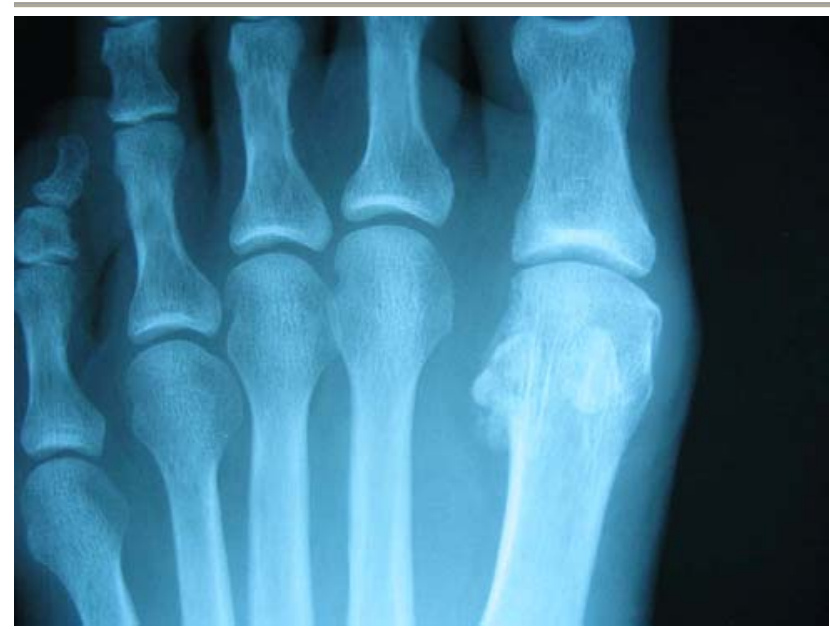

Figure 1 This view shows a comminuted fracture of the fibular sesamoid with sclerotic margins of the bone consistent with avascular necrosis.

\section{Surgical Technique}

A local block was administered using 0.5\% marcaine. The area was cleansed and draped in a sterile manner. A plantar incision was made just lateral to the fibular sesamoid. Soft tissue attachment to the sesamoid was sharply dissected with a \#15 blade. (Fig. 2) Care was taken to avoid all neurovascular structures. The entire fibular sesamoid was then excised. The MPJ was visualized and inspected after sesamoid removal. The head was irregular and the articular cartalige was rough and eroded plantarly. (Fig. 3) After removal of the fibular sesamoid, this articular cartilage was also found to be eroded and the morphology was lobulated. A small drill was used to score the rough area of the fibular sesamoidal groove. Closure was performed in layers.

\section{Discussion}

Complications of sesamoid excision may result in a variety of postoperative problems including hallux varus, hallux valgus, and extensus. ${ }^{12}$

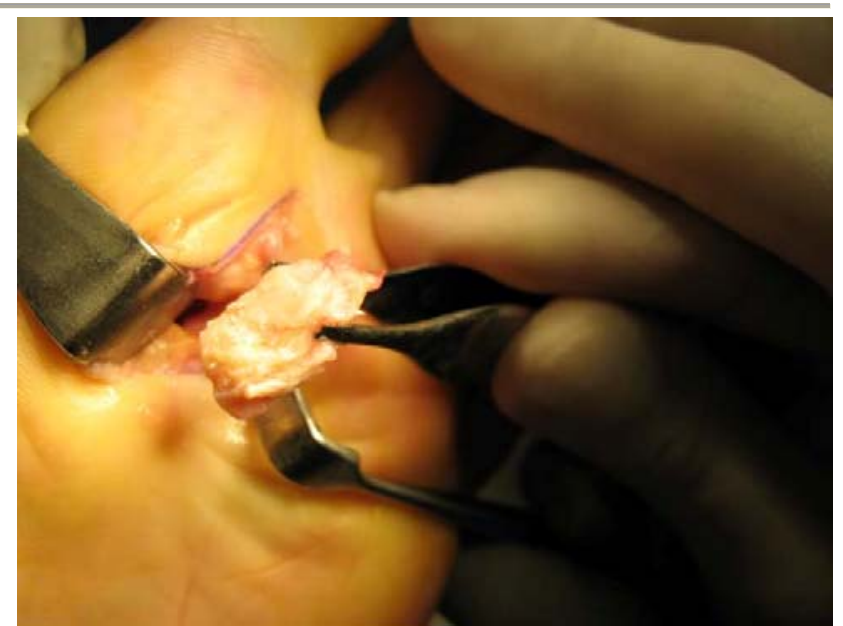

Figure 2 Removal of oversized-fractured sesamoid. Here, plantar bone growth and calcificiation can be seen adding to the patient's painful symptoms.

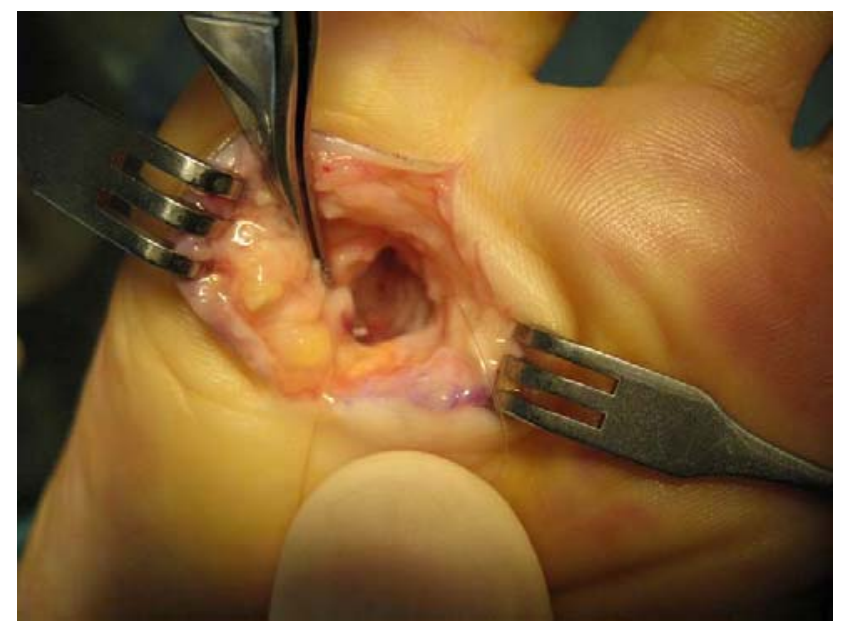

Figure 3 Once the fibular sesamoid is removed, articular erosion is visualized along the metatarsal head. This region can be scored with a k-wire to promote fibrocartilage repair.

Fibular sesamoid excision can result in a hallux varus or valgus in up to $10 \%$ of cases, so Milia, et al., suggest soft tissue repair including preservation of all capsular attachments after excision of the sesamoid to fill in the defect created by removal. ${ }^{6}$ 
In our experience the defect created by removal of the sesamoid was adequately resolved by a standard technique of closure in layers.

Cadaveric study revealed that fibular sesamoid resection led to a decrease in the moment arm of FHL tendon. After resection, extension of the toe caused the flexor tendon to move closer toward the center of joint rotation. ${ }^{1}$

This is important to keep in mind when counseling a patient on postoperative complications and expectations. The decreased distance from line of force to the center of the joint decreases the muscle's mechanical advantage meaning that after surgery the patient may not have full hallux flexion strength. Another study found that by removing only two thirds of the sesamoid, all ligamentous structures would remain intact which may allow the flexor tendons to maintain their moment arm. ${ }^{9}$

Anatomically the sesamoids are contained in the tendon of the flexor hallucis brevis and articulate with the first metatarsal head in sagittally oriented grooves separated by a central ridge or christa. The lateral groove is oriented in a more vertical position than the medial. ${ }^{11}$

Ossification of the sesamoid occurs during the eighth year and multiple centers of ossification can be observed. ${ }^{11}$ They may vary in size and morphology however. In the largest study of sesamoid morphology, Karadaglis and Grace found that sesamoid bones are equal in size in $50 \%$ of feet, the tibial sesamoid is bigger in 35\%, and the fibular sesamoid in 15\%. The shape was characterized as normal, partite, or hour glass. They also report on many patterns of sesamoid partition. $^{5}$

Incision has been described dorsally and plantarly. Advantage of a plantar incision is the close proximity to the sesamoid, however there is a higher risk of injury to the flexor hallucis longus tendon and the neurovascular bundle.
Care must be taken not to cut laterally so as to avoid the adductor hallucis. Cutting this tendon may result in a hallux varus deformity. The dorsal incision may be indicated in patients prone to keloid formation or hypertrophic scarring. ${ }^{11}$ Saxena found that the plantar incision had a earlier return to activity time versus the dorsal incision. The planter incision allowed for direct visualization of the tendon and the plantar digital nerve to the hallux, while the dorsal incisions had a higher rate of complication including nerve entrapment in two cases. ${ }^{10}$ This finding is paralleled in other studies as well.

The condition of avascular necrosis (AVN) of the sesamoid was originally described by Axel Reander in $1924 .{ }^{8}$ AVN is not fully understood but is proposed to be the result of chronic micro trauma which disturbs the blood flow to a bone leading to necrosis. ${ }^{4}$ One study by Pretterklieber shows that blood supply to the sesamoids may be provided by one, two or three arteries, and that women had a higher rate of decreased vascular supply to the tibial sesamoid. ${ }^{7}$ Radiographic findings include fragmentation, demineralization, and striped sclerosis. MRI is the most sensitive diagnostic tool which will reveal sclerotic areas and low signal intensity changes on T2 weighted image. This is the best tool to distinguish between stress fracture and AVN. ${ }^{11} \mathrm{~A}$ stress fracture on MRI will reveal bone marrow edema which may also be present in AVN, but without the low signal intensity changes. ${ }^{2}$

Another possible etiology for sesamoid pain is arthritis of the sesamoid. Although the current literature mentions arthritis as a cause of sesamoid pain, the paucity of case studies and research reveals a subject that would benefit from further investigation. The reason so little attention has been paid to this subject seems to be due to the fact that treatment for localized sesamoid pain is the same regardless of the etiology. 
Our patient had the clinical findings typical of fibular sesamoid AVN including radiologic fragmentation and sclerotic margins of the sesamoid with areas of radiolucency and areas of patchy, increased density.

The localization of her pain to the sub first MPJ and the first interspace were good indication that the source of the pain was the fibular seamoid. After inspection of the sesamoid and the MPJ, it was evident that a joint destructive process occurred. Whether the cause of joint erosion is simply arthritis or an articular fracture, the treatment for either is the removal of the painful and dysfunctional sesamoid.

Prognosis of sesamoid resection is good. Milia, et al., showed that a few patients were not fully satisfied with their results due to an incomplete resolution of pain. However, all patients stated they would not have changed their decision to have surgery. ${ }^{6}$ Saxena, et al., showed that twenty of twenty-four patients were able to return to desired activity and no patients had subsequent trauma to the adjacent sesamoid. Return to athletic activity at full strength after fibular sesamoid resection occurred at average of 9.3 weeks, with one patient who returned at 7.3 weeks. ${ }^{10}$ Overall, the fibular sesamoidectomy procedure seems to be highly efficacious after all conservative therapy has failed and when the pain is isolated to the fibular sesamoid.

\section{References}

1. Aper RL, et al.: The effect of hallux sesamoid excison on the flexor hallucis longus moment arm. Clin Orthop Relat Res 325:209-17, 1996.

2. Brandser EA, Buckwalter JA: Imaging studies for diagnosing stress and insufficiency fractures. Iowa Orthop J 16:70-8, 1996.

3. Inge GA, Fergason AB: Surgery of the seamoid bones of the great toe. Arch Surg 27:466-89,1933.

4. Kalweit M, Frank D: Aseptic necrosis of the first metatarsal sesamoid (Morbus Renander) FussSprungg 1:148-151, 2003.

5. Karadaglis D, Grace D: Morphology of the hallux sesamoids. Foot and Ankle Surgery 9 (3),165-167, 2003.

[Abstract]

6. Milia M, Cohen BE, Anderson RB: Plantar approach for isolated fibular hallux sesamoidectomy. Techniques in foot and Ankle Surgery 2(4):268-71, 2003.

7. Pretterklierber ML: Dimensions and arterial vascular supply of the sesamoid bones of the human hallux. Acta Anat. 139:86-90, 1990.

8. Renander A: Two cases of typical osteochondropathy of the medial sesamoid bone of the first metatarsal. Acta Radiol 3:521-527, 1924.

9. Richardson EG: Hallucal sesamoid pain: causes and surgical treatment. J Am Acad Orthop Surg 7(4): 270-278, 1999.

10. Saxena A, Krisdakumtorn T: Return to activity after sesamoidectomy in athletically active individuals. Foot Ankle Int 24(5):415-9, 2003.

11. Waizy H, Jäger M, Abbara-Czardybon M, Schmidt TG, Frank D: Surgical treatment of AVN of the fibular (lateral) sesamoid. Foot Ankle Int 29(2):231-6, 2008.

12. Vanore JV, Jeffrey C, Christensen SR, Kravitz M. Schuberth JL, et al. Clinical Practice Guideline First Metatarsophalangeal Joint Disorders Panel, Diagnosis and Treatment of First Metatarsophalangeal Joint Disorders. Section 4: Sesamoid Disorders. J Foot Ankle Surg. 42:143147, 2003. [Abstract] 